OPEN ACCESS

Edited by:

Panagiotis Drakopoulos, University Hospital Brussels, Belgium

Reviewed by:

Cinzia Antognelli,

University of Perugia, Italy

Andrea Garolla,

University of Padua, Italy

${ }^{*}$ Correspondence:

Enwu Yuan

diyudeshouhuzhe@126.com

Junije Liu

13937112492@126.com

Specialty section:

This article was submitted to

Reproduction,

a section of the journa

Frontiers in Endocrinology

Received: 27 October 2021 Accepted: 13 December 2021

Published: 04 January 2022

Citation:

Dai Y, Liu J, Yuan E, Li Y, Shi Y and Zhang L (2022) Relationship Among Traditional Semen Parameters, Sperm DNA Fragmentation, and Unexplained Recurrent Miscarriage: A Systematic Review and Meta-Analysis.

Front. Endocrinol. 12:802632. doi: 10.3389/fendo.2021.802632

\section{Relationship Among Traditional Semen Parameters, Sperm DNA Fragmentation, and Unexplained Recurrent Miscarriage: A Systematic Review and Meta-Analysis}

\author{
Yanpeng Dai ${ }^{1}$, Junjie Liu ${ }^{2 *}$, Enwu Yuan ${ }^{1 *}$, Yushan $L_{i}{ }^{2}$, Ying Shi ${ }^{1}$ and Linlin Zhang ${ }^{1}$ \\ ${ }^{1}$ Department of Clinical Laboratory, The Third Affiliated Hospital of Zhengzhou University, Zhengzhou, China, ${ }^{2}$ Henan Human \\ Sperm Bank, The Third Affiliated Hospital of Zhengzhou University, Zhengzhou, China
}

Several studies have explored the relationship among traditional semen parameters, sperm DNA fragmentation (SDF), and unexplained recurrent miscarriage (RM); however, the findings remain controversial. Hence, we conducted a meta-analysis to explore the relationship among traditional semen parameters, SDF, and unexplained RM. Multiple databases, including PubMed, Google Scholar, MEDLINE, Embase, Cochrane Library, Web of Science, and China National Knowledge Infrastructure (CNKI), were searched to identify relevant publications. From the eligible publications, data were extracted independently by two researchers. A total of 280 publications were identified using the search strategy. According to the inclusion/exclusion criteria, 19 publications were eligible. A total of 1182 couples with unexplained RM and 1231 couples without RM were included in this meta-analysis to assess the relationship among traditional semen parameters, SDF, and unexplained RM. Our results showed that couples with unexplained RM had significantly increased levels of SDF and significantly decreased levels of total motility and progressive motility compared with couples without RM, although significant differences were not observed in the semen volume, sperm concentration, and total sperm count between couples with and without RM. The SDF assay may be considered for inclusion in evaluations of couples with unexplained RM.

Keywords: DNA fragmentation, semen quality, recurrent miscarriage, sperm, meta-analysis

\section{INTRODUCTION}

A uniform definition of recurrent miscarriage (RM) has not been established. The American Society for Reproductive Medicine (ASRM) defines RM as two or more consecutive miscarriages (1), while the Royal College of Obstetricians and Gynecologists (RCOG), the Chinese Society of Obstetrics and Gynecology, and the European Society of Human Reproduction and Embryology (ESHRE) guidelines define RM as three or more consecutive miscarriages (2-4). RM affects approximately $1 \%$ of couples trying to conceive (5). In almost half of the cases of RM, the etiology of the affected 
couples remains unclear (1). Research has mainly focused on female factors for RM, but the role of male factors in RM has recently gained attention (6-8).

Male fertility is usually assessed by the semen volume, sperm concentration, total sperm count, progressive motility, and total motility according to WHO guidelines. However, traditional semen parameters have relatively poor predictive value for spermatozoa fertilizing capacity and reproductive outcomes (9). The integrity of sperm DNA is essential for the accurate transmission of genetic information from father to offspring. Sperm DNA fragmentation (SDF) is used to assess the integrity of sperm chromatin and has been increasingly recognized as crucial because of its diagnostic potential in terms of male fertility and pregnancy outcomes. There are three main hypotheses regarding the molecular mechanism of sperm DNA damage, including oxidative stress: chromatin packaging abnormalities, and apoptosis (10). A certain degree of sperm DNA damage can be repaired by the oocyte; however, when the damage exceeds the repair capacity of the oocyte, then adverse pregnancy outcomes may occur (11). Many clinical studies have investigated the relationship between SDF and reproductive outcomes, and several studies have suggested that SDF is associated with poor fertilization, suboptimal embryo quality, and lower pregnancy rates (12-15). Gandini et al. suggest that sperm with DNA damage are capable of fertilizing an oocyte (16). However, other studies have indicated that SDF is not associated with the fertilization rate or pregnancy outcome $(17,18)$. Thus, the implications of SDF on fertilization rate and pregnancy outcome remain controversial.

Many SDF assays have been developed, and the main methods are as follows: sperm chromatin dispersion (SCD) (19-23), terminal deoxyuridine nick end labeling (TUNEL) (24-28), acridine orange test (AOT) (29), sperm chromatin structure assay (SCSA) (27, 30-35), and aniline blue (AB) staining (36). TUNEL is a direct method of measuring single and double DNA strand breaks by using probes, while SCD, SCSA, AOT, and AB staining are indirect methods that use the increased susceptibility of sperm DNA damage to acidinduced denaturation.

This systematic review and meta-analysis aimed to assess the relationship among traditional semen parameters, SDF, and unexplained RM.

\section{MATERIALS AND METHODS}

\section{Literature Search}

The study was performed in accordance with the Preferred Reporting Items for Systematic Reviews and Meta-Analyses (PRISMA) guidelines (37). Multiple databases, including PubMed, Google Scholar, Cochrane Library, Embase, Web of Science, MEDLINE, and China National Knowledge Infrastructure (CNKI), were searched to identify relevant articles from inception to October 2021. The search was limited to human studies published in English and included using the following terms: "recurrent pregnancy loss", "repeated pregnancy loss", "recurrent abortions", "recurrent spontaneous abortion", "recurrent miscarriage", "sperm DNA fragmentation", "sperm DNA integrity", "sperm DNA damage", "SDF", "DFI", "traditional semen parameters", and "conventional semen parameters".

\section{Selection Criteria}

Studies that met the following criteria were included in this study: (1) original research; (2) the topic is unexplained RM; (3) natural conception; and (4) the data for traditional semen parameters and SDF are expressed as the means with standard deviations (SDs). The exclusion criteria were as follows: (1) reviews, letters, editorials, and abstracts; (2) inaccessible full articles; (3) case-only studies; and (4) duplicate publications.

\section{Selection of Publications}

Based on the predefined inclusion/exclusion criteria, all publications were independently selected for eligibility by two authors (Y.D. and J.L.). After removing duplicates, articles were selected by reviewing the titles and abstracts. The remaining publications were retrieved for full-text assessment if their appropriateness could not be determined. Any discrepancy was resolved through discussion with the third reviewer (E.Y.).

\section{Data Extraction}

From the eligible publications, data were extracted independently by two authors (Y.D. and J.L.). Any discrepancy between the two authors (Y.D. and J.L.) was resolved by discussion with the third reviewer (E.Y.). The following information was collected for each eligible publication: name of the first author, publication year, country of origin, ethnicity group, type of study design, sample size, and methods used to evaluate SDF. The main characteristics of the included studies are listed in Table $\mathbf{1}$.

\section{Quality Assessment of the Included Publications}

Quality assessments were performed using the NewcastleOttawa Scale (NOS) (39). A NOS score of $\geq 6$ was considered high quality (40).

\section{Statistical Analysis}

All analyses were performed using Stata/SE 12.0 (StataCorp, College Station, Texas, USA). The heterogeneity between publications was calculated using the $\mathrm{I}^{2}$ statistic and Cochran's $\mathrm{Q}$ test. Heterogeneity was considered significant at $P<0.10$ and/or $I^{2}<50 \%$. Based on the heterogeneity assessment, random- or fixed-effects models were selected to calculate the weighted mean differences (WMDs) and their corresponding 95\% confidence intervals (CIs). To explore the potential sources of heterogeneity, subgroup analyses were performed. To estimate the stability of the pooled results, a sensitivity analysis was conducted by excluding each publication. To estimate the possible publication bias, Egger's regression test and Begg's funnel plot were used. Statistical significance was set at $P<0.05$. 
TABLE 1 | Main characteristics of the included studies in the meta-analysis.

\begin{tabular}{|c|c|c|c|c|c|c|c|c|c|}
\hline Author (year) & Country & Ethnicity & $\begin{array}{l}\text { Study } \\
\text { design }\end{array}$ & Cases & Controls & $\begin{array}{c}\text { Sample size Cases/ } \\
\text { controls }\end{array}$ & $\begin{array}{l}\text { Samples for } \\
\text { DFI }\end{array}$ & Assay & $\begin{array}{l}\text { Quality } \\
\text { score }\end{array}$ \\
\hline Absalan et al. (19) & Iran & Asian & Prospective & $\begin{array}{l}\mathrm{RPL} \geq 3 \\
\text { times }\end{array}$ & Fertile & $30 / 30$ & Fresh semen & SCD & 7 \\
\hline Bareh et al. (24) & USA & Caucasian & Prospective & $\begin{array}{l}\mathrm{RPL} \geq \\
\text { 2times }\end{array}$ & $\geq 1$ live birth & $26 / 31$ & Fresh semen & TUNEL & 7 \\
\hline $\begin{array}{l}\text { Bhattacharya et al. } \\
\text { (29) }\end{array}$ & India & Asian & Prospective & $\begin{array}{l}\mathrm{RPL} \geq \\
\text { 2times }\end{array}$ & $\geq 1$ live birth & $74 / 65$ & Fresh semen & AOT & 7 \\
\hline Brahem et al. (25) & Tunisia & African & Prospective & $\begin{array}{l}\mathrm{RPL} \geq \\
\text { 2times }\end{array}$ & Fertile & $31 / 20$ & Frozen semen & TUNEL & 7 \\
\hline Carlini et al. (26) & Italy & Caucasian & Prospective & $\begin{array}{l}\mathrm{RPL} \geq \\
\text { 2times }\end{array}$ & $\geq 1$ live birth & $112 / 114$ & Fresh semen & TUNEL & 8 \\
\hline Carrell et al. (38) & USA & Caucasian & Prospective & $\begin{array}{l}\mathrm{RPL} \geq 3 \\
\text { times }\end{array}$ & $\geq 1$ live birth & $21 / 26$ & Frozen semen & TUNEL & 7 \\
\hline Coughlan et al. (20) & UK & Caucasian & Prospective & $\begin{array}{l}\mathrm{RPL} \geq 3 \\
\text { times }\end{array}$ & $\geq 1$ live birth & $16 / 7$ & Fresh semen & SCD & 8 \\
\hline Eisenberg et al. (30) & USA & Caucasian & Prospective & $\begin{array}{l}\mathrm{RPL} \geq \\
\text { 2times }\end{array}$ & $\begin{array}{l}\text { Currently } \\
\text { pregnant }\end{array}$ & $14 / 246$ & Frozen semen & SCSA & 9 \\
\hline Gil-villa et al. (31) & USA & Caucasian & Prospective & $\begin{array}{l}\mathrm{RPL} \geq \\
\text { 2times }\end{array}$ & $\geq 1$ live birth & $23 / 11$ & Frozen semen & SCSA & 7 \\
\hline Imam et al. (32) & India & Asian & Retrospective & $\begin{array}{l}\mathrm{RPL} \geq 3 \\
\text { times }\end{array}$ & $\geq 1$ live birth & $20 / 20$ & Frozen semen & SCSA & 8 \\
\hline Kamkar et al. (27) & Iran & Asian & Retrospective & $\begin{array}{l}\mathrm{RPL} \geq \\
\text { 2times }\end{array}$ & $\geq 1$ live birth & $42 / 42$ & Frozen semen & $\begin{array}{l}\text { SCSA and } \\
\text { TUNEL }\end{array}$ & 7 \\
\hline Khadem et al. (21) & Iran & Asian & Prospective & $\begin{array}{l}\mathrm{RPL} \geq 3 \\
\text { times }\end{array}$ & $\begin{array}{l}\text { Currently } \\
\text { pregnant }\end{array}$ & $30 / 30$ & Fresh semen & SCD & 8 \\
\hline Kumar et al. (33) & India & Asian & Prospective & $\begin{array}{l}\mathrm{RPL} \geq 3 \\
\text { times }\end{array}$ & $\geq 1$ live birth & $45 / 20$ & Frozen semen & SCSA & 7 \\
\hline $\begin{array}{l}\text { Ribas-Maynou et al. } \\
\text { (22) }\end{array}$ & Spain & Caucasian & Prospective & $\begin{array}{l}\mathrm{RPL} \geq \\
\text { 2times }\end{array}$ & $\geq 1$ live birth & $20 / 25$ & Frozen semen & SCD & 8 \\
\hline Ruixue et al. (36) & China & Asian & Prospective & $\begin{array}{l}\mathrm{RPL} \geq 3 \\
\text { times }\end{array}$ & $\begin{array}{l}\text { Currently } \\
\text { pregnant }\end{array}$ & $68 / 63$ & Fresh semen & AB staining & 7 \\
\hline Venkatesh et al. (34) & India & Asian & Prospective & $\begin{array}{l}\mathrm{RPL} \geq \\
\text { 3times }\end{array}$ & $\geq 1$ live birth & $16 / 20$ & Frozen semen & SCSA & 7 \\
\hline Zhang et al. (23) & China & Asian & Prospective & $\begin{array}{l}\mathrm{RPL} \geq \\
\text { 2times }\end{array}$ & $\geq 1$ live birth & $111 / 30$ & Fresh semen & SCD & 7 \\
\hline Zhu et al. (35) & China & Asian & Retrospective & $\begin{array}{l}\mathrm{RPL} \geq \\
\text { 2times }\end{array}$ & Fertile & $461 / 411$ & Fresh semen & SCSA & 8 \\
\hline Zidi-Jrah et al. (28) & Tunisia & African & Prospective & $\begin{array}{l}\mathrm{RPL} \geq \\
\text { 2times }\end{array}$ & $\geq 1$ live birth & $22 / 20$ & Frozen semen & TUNEL & 7 \\
\hline
\end{tabular}

SCSA, sperm chromatin structure assay; SCD, sperm chromatin dispersion; TUNEL, terminal TdT-mediated dUTP-nick-end labeling; AOT, acridine orange test; AB staining, aniline blue staining.

\section{RESULTS}

\section{Selection of Publications}

Figure 1 shows the selection process of eligible publications. Based on our search strategy, 280 publications were initially identified through a database search. A total of 249 titles and abstracts of publications were reviewed after removing 31 duplicates. After screening the titles and abstracts of publications, 26 potentially relevant publications were found. The remaining publications were retrieved for full-text assessment. After full-text assessment of the remaining publications, 7 publications were excluded for various reasons. A total of 19 publications were finally included in the metaanalysis, which involved 2413 subjects (1182 couples with unexplained RM and 1231 couples without RM).

\section{Characteristics of the Eligible Publications}

Table 1 presents the main characteristics of the eligible publications. All included articles were of relatively high quality. None of the couples had received assisted reproductive treatments. Male partners of couples without unexplained RM had proven natural fertility with one or more live births. Female partners were under 40 years of age in all eligible studies. Female partners had normal ovarian function and a normal uterus (demonstrated by hysteroscopy, hysterosalpingography, and/or hysterosonography). Female partners with any of the following were excluded: abnormal karyotypes and uterine structural abnormalities.

\section{Relation Between Traditional Semen Parameters and Unexplained RM}

Sixteen studies explored the relation between traditional semen parameters and unexplained RM. For all eligible studies, the evaluation of traditional semen parameters was performed in fresh semen samples. The pooled results showed that there were no relations between unexplained RM and semen volume ( $\mathrm{WMD}=-0.12,95 \% \mathrm{CI}=-0.32$ to $0.08, P>0.05$ ), sperm concentration ( $\mathrm{WMD}=-2.28,95 \% \mathrm{CI}=-4.58$ to $0.02, P>0.05$ ), and total sperm count $(\mathrm{WMD}=-10.73,95 \% \mathrm{CI}=-22.11$ to 0.66 , 


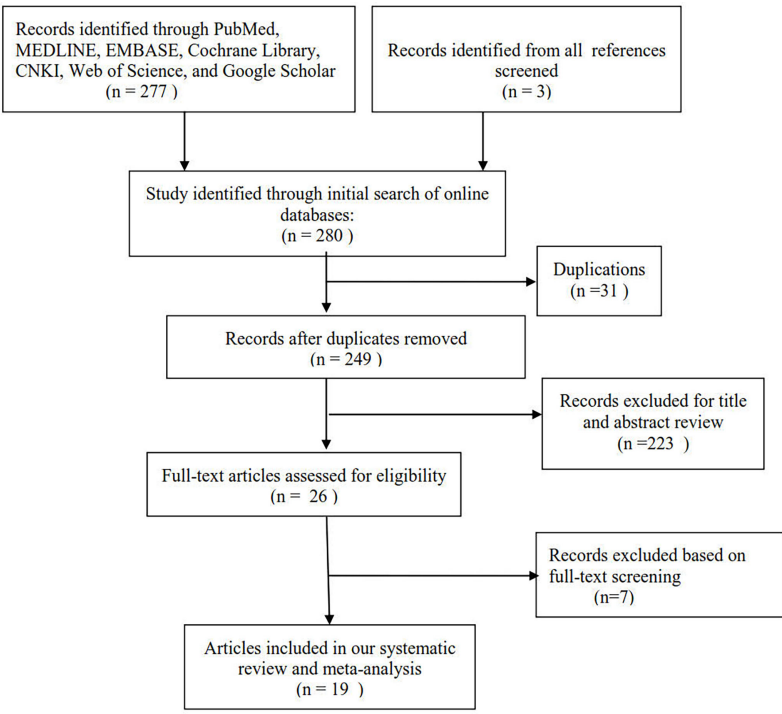

FIGURE 1 | Selection process for eligible publications.

$P>0.05$ ) (Figure 2). However, the pooled results showed that there were significant relations between unexplained RM and progressive motility ( $\mathrm{WMD}=-4.75,95 \% \mathrm{CI}=-8.35$ to -1.15 , $P<0.05)$ and total motility $(\mathrm{WMD}=-10.30,95 \% \mathrm{CI}=-15.03$ to -5.57, $P<0.05)$ (Figure 2). Since significant heterogeneity was observed for the total and progressive motility $\left(I^{2}=82.3 \%\right.$, $\left.P<0.001 ; I^{2}=99.4 \%, P<0.001\right)$, subgroup analyses were performed by the study design type, RM definition, and ethnicity to explore the source of heterogeneity (Figure 2). For the majority of the subgroups, the percentages of total and progressive motility were significantly lower in couples with unexplained RM than in couples without RM (Figures 3A-F).

\section{Relation Between SDF and Unexplained RM}

Seventeen studies explored the relations between SDF and unexplained RM. For 8 of these studies, SDF was assessed using fresh semen samples. The pooled results showed that couples with unexplained RM had significantly increased levels of SDF compared with couples without RM (WMD $=8.45,95 \%$ $\mathrm{CI}=1.48$ to $15.42, P=0.018$ ) (Table 2 and Figure 4). Because significant heterogeneity was observed for SDF $\left(I^{2}=99.4 \%\right.$, $P<0.001)$, subgroup analyses were performed by the assay type, RM definition, and ethnicity to explore the source of heterogeneity (Table 2 and Figure 4). Subgroup analysis by SDF assay also showed a significant association between couples with and without RM for the SCD assay (WMD $=2.15,95 \%$ $\mathrm{CI}=1.62$ to $2.68, P<0.001$ ) (Table 2 and Figure $4 \mathrm{~A}$ ). The subgroup analysis by the definition of RM showed that couples with a history of $\mathrm{RM} \geq 2$ times and $\geq 3$ times had significantly increased levels of SDF compared with couples without RM ( $\mathrm{WMD}=11.22,95 \% \mathrm{CI}=1.26$ to $21.19, P=0.027$ and $\mathrm{WMD}=3.33$, $95 \% \mathrm{CI}=1.20$ to $5.46, P=0.002$ ) (Table 2 and Figure 4B). The subgroup analysis by ethnicity also showed similar results to the overall analysis in the Asian subgroup (WMD $=5.90,95 \%$ $\mathrm{CI}=2.30$ to $9.50, P=0.001$ ) (Table 2 and Figure 4C).

\section{Sensitivity Analyses}

The sensitivity analysis showed that the pooled results were stable and reliable (Figure 5).

\section{Publication Bias}

As shown in Table 2 and Figure 6, our results showed that there was no publication bias for the semen volume, sperm concentration, total sperm count, progressive motility, total motility, and SDF.

\section{DISCUSSION}

RM affects approximately $1 \%$ of couples trying to conceive (5). In almost half of the cases of RM, the etiology of the affected couples remains unclear (1). The role of female factors in RM has been studied intensively, but the role of male factors has been less thoroughly investigated (6-8).

Some studies have reported that male partners of couples with unexplained RM had significantly decreased levels of semen volume (26) and progressive motility (19, 27, 31, 34, 35) compared with couples without RM, but significant differences were not observed in sperm concentration (19-21, 23-31, 33, 35, $36,38)$, total sperm count $(23,24,26,27,29,30)$ and total motility $(20,21,24,36)$ between the two groups. Some studies reported that couples with unexplained RM had significantly increased levels of sperm concentration (34) and total motility (25, 27-29) compared with couples without RM, but significant differences were not observed in semen volume $(21,23,25,27,28,30-35)$ and progressive motility $(23,26,29)$ between the two groups. The combined results of this meta-analysis showed that couples with unexplained RM had significantly decreased levels of progressive motility and total motility than those of couples without RM. The combined results demonstrate that women whose partners had a higher percentage of progressive motility and total motility were more likely to have a successful pregnancy while women whose partners had a lower percentage of progressive motility and total motility were less likely to conceive and/or more likely to experience pregnancy loss.

Marked between-study heterogeneity was observed for progressive motility and total motility, and it could not be ignored. Therefore, subgroup analyses by the study design type, RM definition, and ethnicity were performed to explore the source of heterogeneity. However, heterogeneity was still observed despite performing the subgroup analyses. Such heterogeneity may be explained by differences in age and number of participants, duration of sexual abstinence, ethnicity, lifestyle habits, laboratory techniques, etc.

However, approximately $15 \%$ of male factor infertility patients show normal parameters in their ejaculates (41), suggesting that conventional semen parameters are poor predictors of reproductive outcome and that a definitive diagnosis of male 

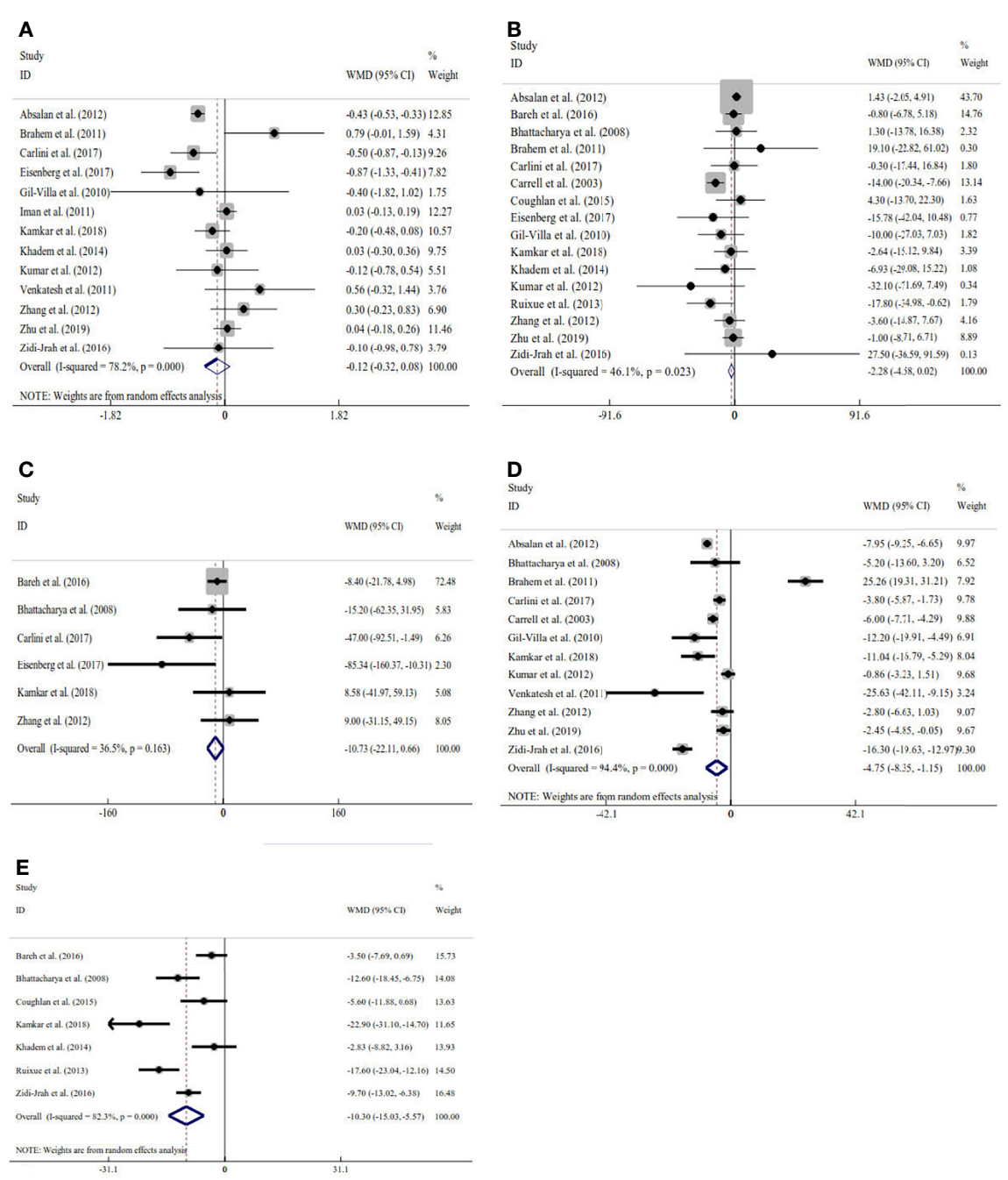

FIGURE 2 | Meta-analysis of the relations between traditional semen parameters and unexplained recurrent miscarriage. (A) Relation between volume and unexplained recurrent miscarriage; (B) relation between sperm concentration and unexplained recurrent miscarriage; (C) relation between total sperm count and unexplained recurrent miscarriage; (D) relation between progressive motility and unexplained recurrent miscarriage; and (E) relation between total motility and unexplained recurrent miscarriage.

infertility cannot be made by a routine semen analysis alone, which is because several factors other than conventional semen parameters affect the fertilization ability of spermatozoa.

Routine semen analysis does not assess all aspects of sperm quality. SDF is used to assess the integrity of sperm chromatin and may be a better predictor of male fertility and reproductive outcomes than conventional semen parameters. Sperm DNA integrity plays an important role in the initiation and maintenance of pregnancy (42). The study of sperm DNA integrity may be important for understanding the pathogenesis of unexplained RM. However, the relationship between sperm DNA integrity and unexplained RM remains controversial. Some studies $(19,20,22,24-29,32,33,35,36,38)$ have reported that couples with unexplained RM had significantly increased levels of SDF compared with those of couples without RM. However, other studies $(23,30,31)$ have reported no significant differences in SDF between couples with and without RM. For these studies, SDF was assessed using fresh or cryopreserved semen samples. The cryopreservation process can alter the sperm quality, particularly the motility and sperm DNA integrity (43-45). Only those studies that assessed SDF with fresh semen samples were included in this meta-analysis to evaluate the relationship between SDF and unexplained RM. The combined results of this meta-analysis demonstrated that couples with unexplained RM had significantly increased levels of SDF compared with couples without RM. Our results demonstrated that women whose partners had a lower percentage of SDF were more likely to have a successful pregnancy while women whose partners had a higher percentage of SDF were more likely to experience pregnancy loss. Our results also suggested that male factors may be involved in the pathogenesis of RM and that SDF might be used as a tool to evaluate the risk of RM. However, future large 

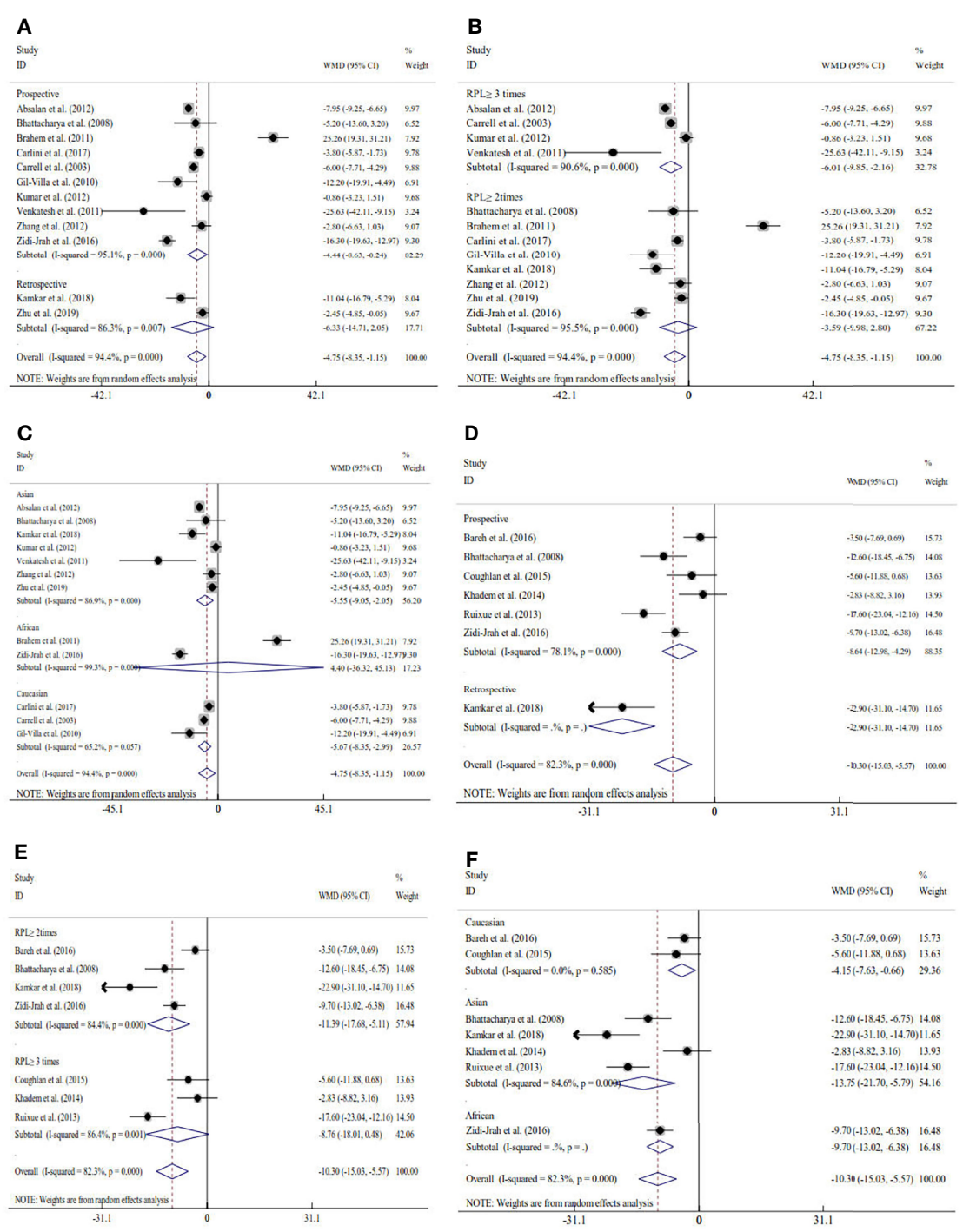

FIGURE 3 | Subgroup analyses for progressive motility by the type of study design (A), definition of recurrent miscarriage (B), and ethnicity (C); subgroup analyses for total motility by type of study design (D), definition of recurrent miscarriage $(\mathbf{E})$, and ethnicity $(\mathbf{F})$.

TABLE 2 | Subgroup analyses by sperm DNA fragmentation assay, definition of recurrent miscarriage, sperm preservation and ethnicity.

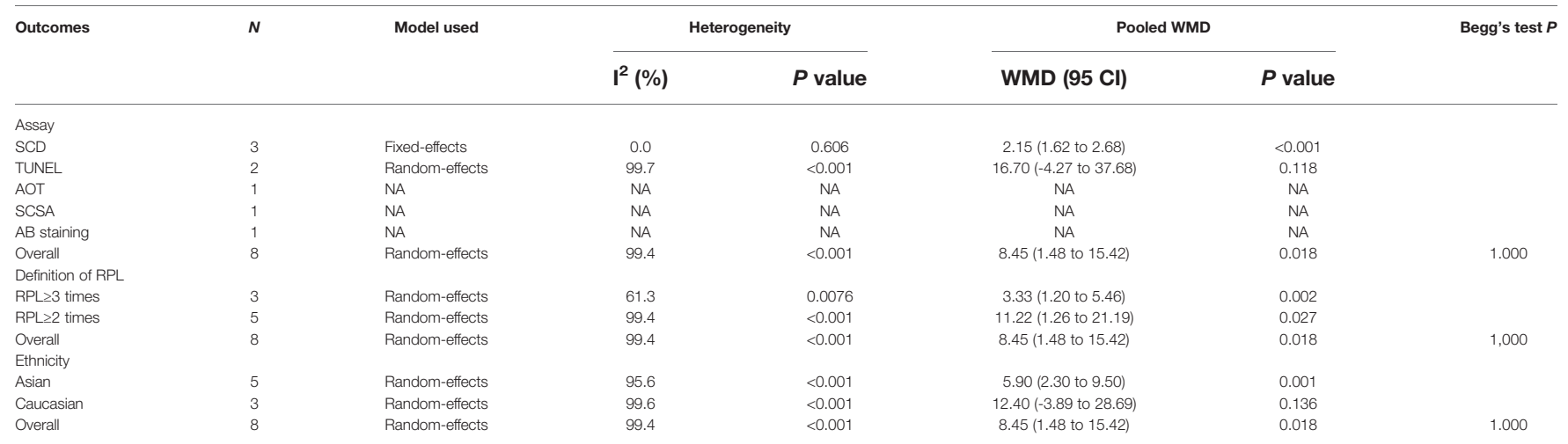


prospective studies are needed to evaluate the impact of elevated SDF on the risk of RM.

Marked between-study heterogeneity was observed, and it could not be ignored. Several factors may account for the measured heterogeneity. First, there are several methods used to assess SDF. Second, there are two definitions of unexplained RM. Third, the subjects included in the studies were of diverse ethnic backgrounds. All of these factors may have significantly affected the between-study heterogeneity. Subgroup analyses by the assay type, RM definition, and ethnicity were performed to explore the source of heterogeneity. However, heterogeneity was still observed despite performing these subgroup analyses. The results of the subgroups by the definition of RM showed that the couples with a history of $\mathrm{RM} \geq 2$ times and $\geq 3$ times had significantly increased levels of SDF. Given the limited sample size of the included studies and the significant heterogeneity between studies, further large prospective cohort studies are needed to validate these findings.
There were four strengths of this meta-analysis. First, more reliable results can be obtained as a result of the large sample size. Second, we also assessed the relation between traditional semen parameters and unexplained RM. Third, the subgroup analyses by the assay type, RM definition, and ethnicity were also conducted in this study. Fourth, no publication bias was found in this meta-analysis.

This meta-analysis has two limitations. First, between-study heterogeneity was found despite using strict inclusion/exclusion criteria. Second, the number of included publications was small in some subgroups.

Couples with unexplained RM had significantly increased levels of SDF compared with couples without RM, and they also had significantly decreased progressive motility and total motility. The SDF assay may be considered for inclusion in evaluations of couples with unexplained RM. Future large prospective studies are needed to evaluate the impact of elevated SDF on the risk of RM.

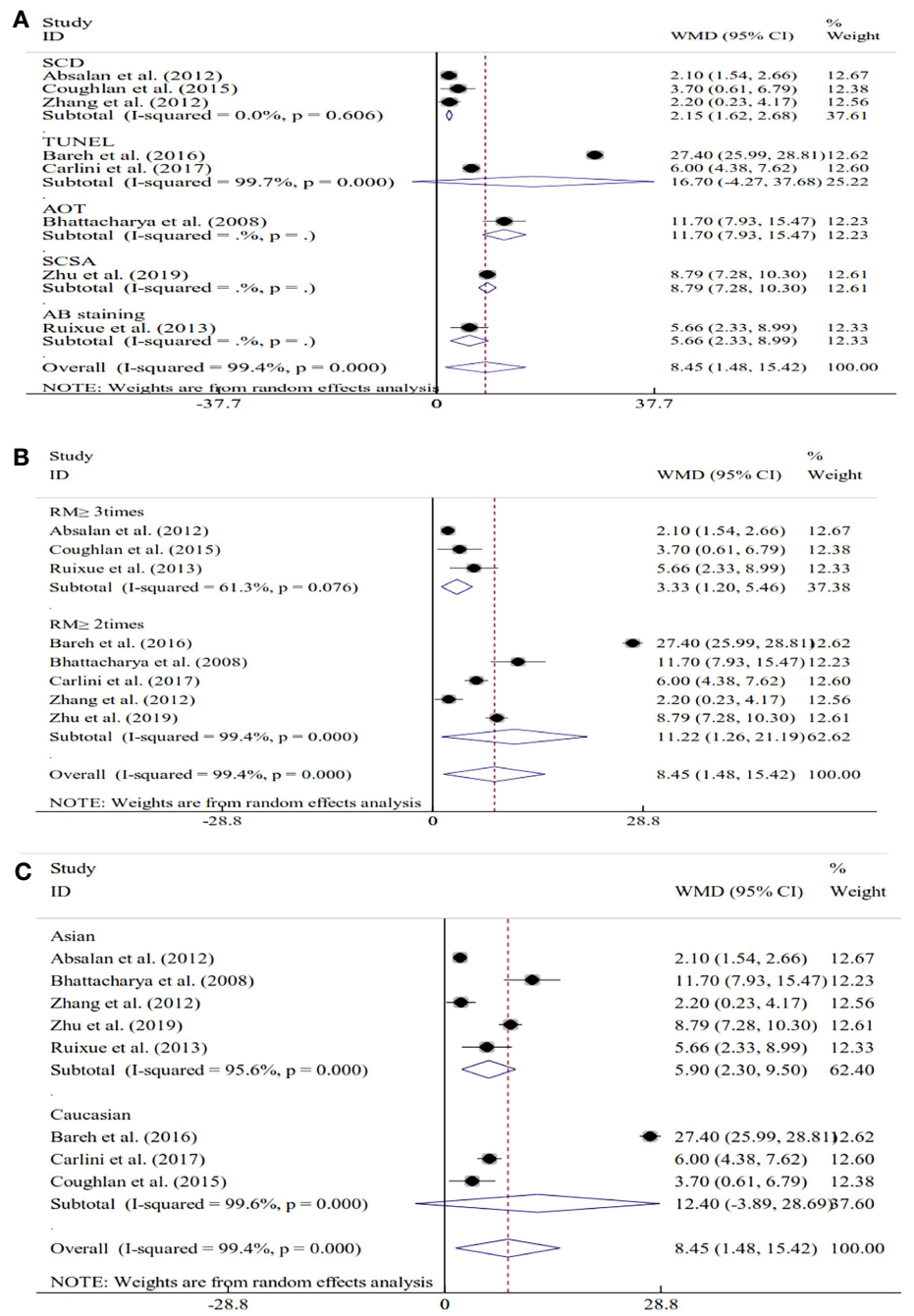

FIGURE 4 | Subgroup analyses based on the sperm DNA fragmentation assay (A), the definition of recurrent miscarriage (B), and ethnicity (C). 
A

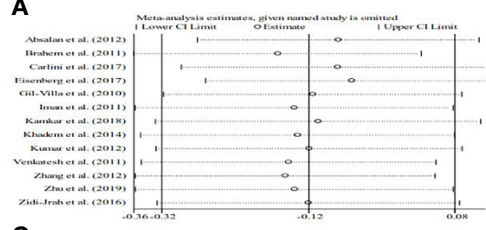

C
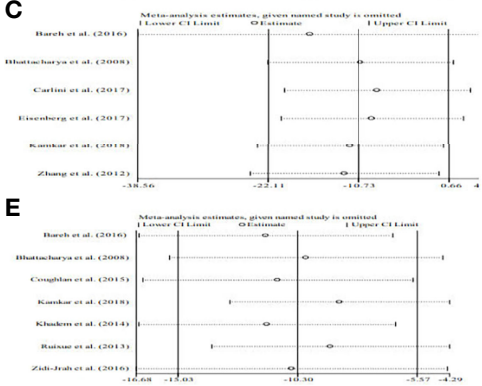
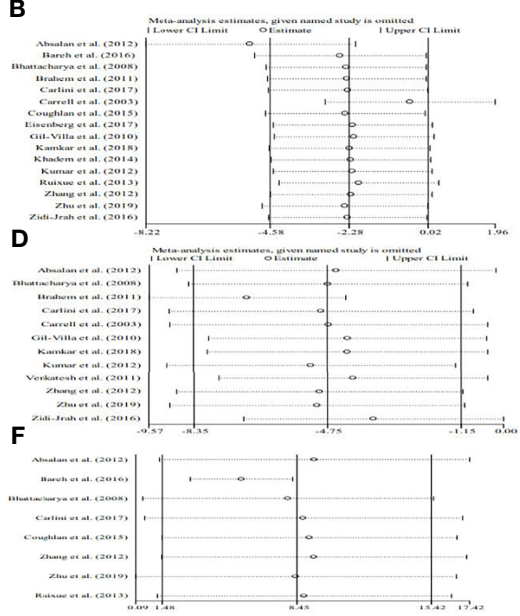

FIGURE 5 | Begg's funnel plot of the relationship among volume (A), sperm concentration (B), total sperm count (C), progressive motility (D), total motility (E), and sperm DNA fragmentation $(\mathbf{F})$ and unexplained recurrent miscarriage.

A

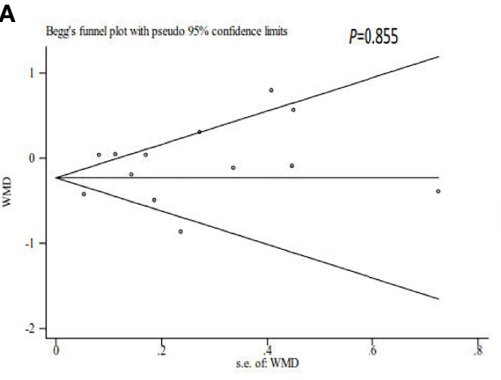

C

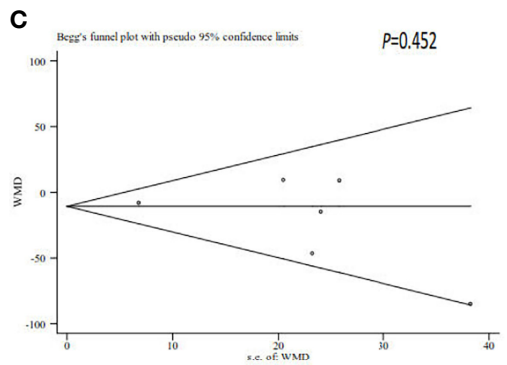

E

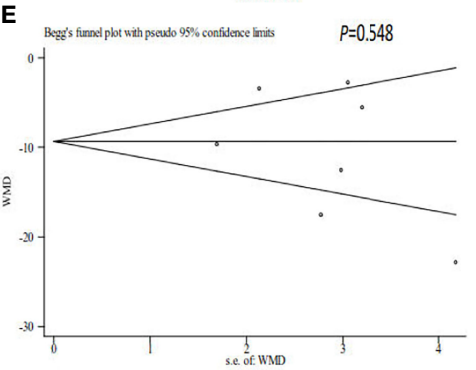

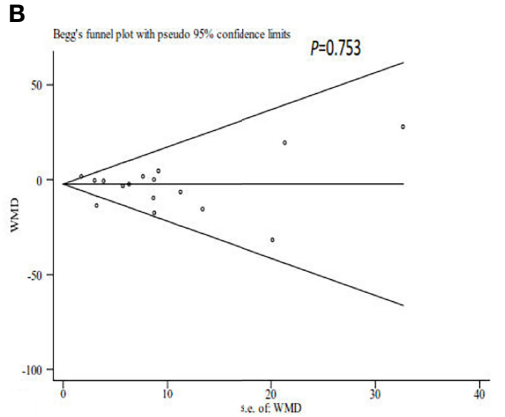

D
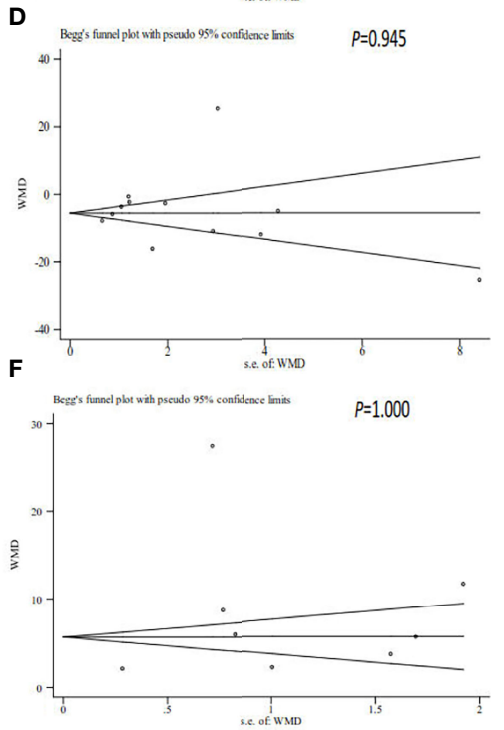

FIGURE 6 | Sensitivity analysis of the relationship among volume (A), sperm concentration (B), total sperm count (C), progressive motility (D), total motility (E), and sperm DNA fragmentation (F) and unexplained recurrent miscarriage. 


\section{DATA AVAILABILITY STATEMENT}

The original contributions presented in the study are included in the article/supplementary material. Further inquiries can be directed to the corresponding authors.

\section{AUTHOR CONTRIBUTIONS}

All authors contributed to the study's conception and design. YD and JL participated in drafting the manuscript and the

\section{REFERENCES}

1. Practice Committee of the American Society for Reproductive Medicine. Evaluation and Treatment of Recurrent Pregnancy Loss: A Committee Opinion. Fertil Steril (2012) 98(5):1103-11. doi: 10.1016/j.fertnstert.2012.06.048

2. Royal College of Obstetricians and Gynecologists. The Investigation and Treatment of Couples With Recurrent First-Trimester and Second-Trimester Miscarriage. RCOG Green-top Guideline (2011) 17:1-18.

3. Obstetrics Subgroup Chinese Society of Obstetrics and Gynecology. Chinese Expert Consensus on the Diagnosis and Tretment of Recurrent Spontaneous Abortion]. Zhonghua Fu Chan Ke Za Zhi (2016) 51(1):3-9. doi: 10.3760/ cma.j.issn.0529-567X.2016.01.002

4. Bender Atik R, Christiansen OB, Elson J, Kolte AM, Lewis S, Middeldorp S, et al. ESHRE Guideline: Recurrent Pregnancy Loss. Hum Reprod Open (2018) 2018(2):1-12. doi: 10.1093/hropen/hoy004

5. Porter TF, Scott JR. Evidence-Based Care of Recurrent Miscarriage. Best Pract Res Clin Obstet Gynaecol (2005) 19(1):85-101. doi: 10.1016/ j.bpobgyn.2004.11.005

6. Kumar N, Singh AK. Trends of Male Factor Infertility, an Important Cause of Infertility: A Review of Literature. J Hum Reprod Sci (2015) 8(4):191-6. doi: 10.4103/0974-1208.170370

7. Puscheck EE, Jeyendran RS. The Impact of Male Factor on Recurrent Pregnancy Loss. Curr Opin Obstet Gynecol (2007) 19(3):222-8. doi: 10.1097/GCO.0b013e32813e3ff0

8. Nanassy L, Carrell DT. Paternal Effects on Early Embryogenesis. J Exp Clin Assist Reprod (2008) 5:2. doi: 10.1186/1743-1050-5-2

9. Lewis SE. Is Sperm Evaluation Useful in Predicting Human Fertility? Reproduction (2007) 134(1):31-40. doi: 10.1530/REP-07-0152

10. Schulte RT, Ohl DA, Sigman M, Smith GD. Sperm DNA Damage in Male Infertility: Etiologies, Assays, and Outcomes. J Assist Reprod Genet (2010) 27 (1):3-12. doi: 10.1007/s10815-009-9359-x

11. Bungum M, Bungum L, Lynch KF, Wedlund L, Humaidan P, Giwercman A. Spermatozoa DNA Damage Measured by Sperm Chromatin Structure Assay (SCSA) and Birth Characteristics in Children Conceived by IVF and ICSI. Int J Androl (2012) 35(4):485-90. doi: 10.1111/j.1365-2605. 2011.01222.x

12. Agarwal A, Majzoub A, Esteves SC, Ko E, Ramasamy R, Zini A. Clinical Utility of Sperm DNA Fragmentation Testing: Practice Recommendations Based on Clinical Scenarios. Transl Androl Urol (2016) 5(6):935-50. doi: 10.21037/ tau.2016.10.03

13. Evenson DP, Wixon R. Data Analysis of Two In Vivo Fertility Studies Using Sperm Chromatin Structure Assay-Derived DNA Fragmentation Index vs. Pregnancy Outcome. Fertil Steril (2008) 90(4):1229-31. doi: 10.1016/ j.fertnstert.2007.10.066

14. Simon L, Lewis SE. Sperm DNA Damage or Progressive Motility: Which One Is the Better Predictor of Fertilization In Vitro? Syst Biol Reprod Med (2011) 57 (3):133-8. doi: 10.3109/19396368.2011.553984

15. Velez de la Calle JF, Muller A, Walschaerts M, Clavere JL, Jimenez C, Wittemer C, et al. Sperm Deoxyribonucleic Acid Fragmentation as Assessed by the Sperm Chromatin Dispersion Test in Assisted Reproductive Technology Programs: Results of a Large Prospective Multicenter Study. Fertil Steril (2008) 90(5):1792-9. doi: 10.1016/j.fertnstert.2007.09.021 study design. EY and YL searched and selected the relevant articles. YS and LZ contributed to the statistical analysis. All authors contributed to the article and approved the submitted version.

\section{FUNDING}

By Joint Construction Project of Henan Medical Science and Technology Research Plan (LHGJ20190396).

16. Gandini L, Lombardo F, Paoli D, Caruso F, Eleuteri P, Leter G, et al. FullTerm Pregnancies Achieved With ICSI Despite High Levels of Sperm Chromatin Damage. Hum Reprod (2004) 19(6):1409-17. doi: 10.1093/ humrep/deh233

17. Niu ZH, Shi HJ, Zhang HQ, Zhang AJ, Sun YJ, Feng Y. Sperm Chromatin Structure Assay Results After Swim-Up Are Related Only to Embryo Quality But Not to Fertilization and Pregnancy Rates Following IVF. Asian J Androl (2011) 13(6):862-6. doi: 10.1038/aja.2011.77

18. Bungum M, Humaidan P, Axmon A, Spano M, Bungum L, Erenpreiss J, et al. Sperm DNA Integrity Assessment in Prediction of Assisted Reproduction Technology Outcome. Hum Reprod (2007) 22(1):174-9. doi: 10.1093/ humrep/del326

19. Absalan F, Ghannadi A, Kazerooni M, Parifar R, Jamalzadeh F, Amiri S. Value of Sperm Chromatin Dispersion Test in Couples With Unexplained Recurrent Abortion. J Assist Reprod Genet (2012) 29(1):11-4. doi: 10.1007/s10815-0119647-0

20. Coughlan C, Clarke H, Cutting R, Saxton J, Waite S, Ledger W, et al. Sperm DNA Fragmentation, Recurrent Implantation Failure and Recurrent Miscarriage. Asian J Androl (2015) 17(4):681-5. doi: 10.4103/1008682X.144946

21. Khadem N, Poorhoseyni A, Jalali M, Akbary A, Heydari ST. Sperm DNA Fragmentation in Couples With Unexplained Recurrent Spontaneous Abortions. Andrologia (2014) 46(2):126-30. doi: 10.1111/and.12056

22. Ribas-Maynou J, Garcia-Peiro A, Fernandez-Encinas A, Amengual MJ, Prada E, Cortes P, et al. Double Stranded Sperm DNA Breaks, Measured by Comet Assay, Are Associated With Unexplained Recurrent Miscarriage in Couples Without a Female Factor. PloS One (2012) 7(9):e44679. doi: 10.1371/ journal.pone.0044679

23. Zhang L, Wang L, Zhang X, Xu G, Zhang W, Wang K, et al. Sperm Chromatin Integrity may Predict Future Fertility for Unexplained Recurrent Spontaneous Abortion Patients. Int J Androl (2012) 35(5):752-7. doi: 10.1111/j.13652605.2012.01276.x

24. Bareh GM, Jacoby E, Binkley P, Chang TC, Schenken RS, Robinson RD. Sperm Deoxyribonucleic Acid Fragmentation Assessment in Normozoospermic Male Partners of Couples With Unexplained Recurrent Pregnancy Loss: A Prospective Study. Fertil Steril (2016) 105(2):329-36 e1. doi: 10.1016/j.fertnstert.2015.10.033

25. Brahem S, Mehdi M, Landolsi H, Mougou S, Elghezal H, Saad A. Semen Parameters and Sperm DNA Fragmentation as Causes of Recurrent Pregnancy Loss. Urology (2011) 78(4):792-6. doi: 10.1016/j.urology.2011.05.049

26. Carlini T, Paoli D, Pelloni M, Faja F, Dal Lago A, Lombardo F, et al. Sperm DNA Fragmentation in Italian Couples With Recurrent Pregnancy Loss. Reprod BioMed Online (2017) 34(1):58-65. doi: 10.1016/j.rbmo.2016.09.014

27. Kamkar N, Ramezanali F, Sabbaghian M. The Relationship Between Sperm DNA Fragmentation, Free Radicals and Antioxidant Capacity With Idiopathic Repeated Pregnancy Loss. Reprod Biol (2018) 18(4):330-5. doi: 10.1016/j.repbio.2018.11.002

28. Zidi-Jrah I, Hajlaoui A, Mougou-Zerelli S, Kammoun M, Meniaoui I, Sallem A, et al. Relationship Between Sperm Aneuploidy, Sperm DNA Integrity, Chromatin Packaging, Traditional Semen Parameters, and Recurrent Pregnancy Loss. Fertil Steril (2016) 105(1):58-64. doi: 10.1016/ j.fertnstert.2015.09.041 
29. Bhattacharya SM. Association of Various Sperm Parameters With Unexplained Repeated Early Pregnancy Loss-Which Is Most Important? Int Urol Nephrol (2008) 40(2):391-5. doi: 10.1007/s11255-007-9282-y

30. Eisenberg ML, Sapra KJ, Kim SD, Chen Z, Buck Louis GM. Semen Quality and Pregnancy Loss in a Contemporary Cohort of Couples Recruited Before Conception: Data From the Longitudinal Investigation of Fertility and the Environment (LIFE) Study. Fertil Steril (2017) 108(4):613-9. doi: 10.1016/ j.fertnstert.2017.07.008

31. Gil-Villa AM, Cardona-Maya W, Agarwal A, Sharma R, Cadavid A. Assessment of Sperm Factors Possibly Involved in Early Recurrent Pregnancy Loss. Fertil Steril (2010) 94(4):1465-72. doi: 10.1016/j.fertnstert. 2009.05.042

32. Imam SN, Shamsi MB, Kumar K, Deka D, Dada R. Idiopathic Recurrent Pregnancy Loss: Role of Paternal Factors; A Pilot Study. J Reprod Infertil (2011) 12(4):267-76.

33. Kumar K, Deka D, Singh A, Mitra DK, Vanitha BR, Dada R. Predictive Value of DNA Integrity Analysis in Idiopathic Recurrent Pregnancy Loss Following Spontaneous Conception. J Assist Reprod Genet (2012) 29(9):861-7. doi: 10.1007/s10815-012-9801-3

34. Venkatesh S, Thilagavathi J, Kumar K, Deka D, Talwar P, Dada R. Cytogenetic, Y Chromosome Microdeletion, Sperm Chromatin and Oxidative Stress Analysis in Male Partners of Couples Experiencing Recurrent Spontaneous Abortions. Arch Gynecol Obstet (2011) 284 (6):1577-84. doi: 10.1007/s00404-011-1990-y

35. Zhu XB, Chen Q, Fan WM, Niu ZH, Xu BF, Zhang AJ. Sperm DNA Fragmentation in Chinese Couples With Unexplained Recurrent Pregnancy Loss. Asian J Androl (2019) 22(3):296-301. doi: 10.4103/aja.aja_60_19

36. Ruixue W, Hongli Z, Zhihong Z, Rulin D, Dongfeng G, Ruizhi L. The Impact of Semen Quality, Occupational Exposure to Environmental Factors and Lifestyle on Recurrent Pregnancy Loss. J Assist Reprod Genet (2013) 30 (11):1513-8. doi: 10.1007/s10815-013-0091-1

37. Moher D, Liberati A, Tetzlaff J, Altman DG. Preferred Reporting Items for Systematic Reviews and Meta-Analyses: The PRISMA Statement. BMJ (2009) 339:b2535. doi: 10.1136/bmj.b2535

38. Carrell DT, Liu 1, Peterson CM, Jones KP, Hatasaka HH, Erickson L, et al. Sperm DNA Fragmentation Is Increased in Couples With Unexplained Recurrent Pregnancy Loss. Arch Androl (2003) 49(1):49-55. doi: 10.1080/ 01485010290099390
39. Stang A. Critical Evaluation of the Newcastle-Ottawa Scale for the Assessment of the Quality of Nonrandomized Studies in Meta-Analyses. Eur J Epidemiol (2010) 25(9):603-5. doi: 10.1007/s10654-010-9491-Z

40. Deng C, Li T, Xie Y, Guo Y, Yang QY, Liang X, et al. Sperm DNA Fragmentation Index Influences Assisted Reproductive Technology Outcome: A Systematic Review and Meta-Analysis Combined With a Retrospective Cohort Study. Andrologia (2019) 51(6):13263. doi: 10.1111/and.13263

41. Agarwal A, Allamaneni SS. Sperm DNA Damage Assessment: A Test Whose Time has Come. Fertil Steril (2005) 84(4):850-3. doi: 10.1016/j.fertnstert.2005.03.080

42. Agarwal A, Zini A, Sigman M. Is Sperm DNA Integrity Assessment Useful? J Urol (2013) 190(5):1645-7. doi: 10.1016/j.juro.2013.08.004

43. Raad G, Lteif L, Lahoud R, Azoury J, Azoury J, Tanios J, et al. Cryopreservation Media Differentially Affect Sperm Motility, Morphology and DNA Integrity. Andrology (2018) 6(6):836-45. doi: 10.1111/andr.12531

44. Lusignan MF, Li X, Herrero B, Delbes G, Chan PTK. Effects of Different Cryopreservation Methods on DNA Integrity and Sperm Chromatin Quality in Men. Andrology (2018) 6(6):829-35. doi: 10.1111/andr.12529

45. Zribi N, Chakroun N, Ben Abdallah F, Elleuch H, Sellami A, Gargouri J, et al. Effect of Freezing-Thawing Process and Quercetin on Human Sperm Survival and DNA Integrity. Cryobiology (2012) 65(3):326-31. doi: 10.1016/ j.cryobiol.2012.09.003

Conflict of Interest: The authors declare that the research was conducted in the absence of any commercial or financial relationships that could be construed as a potential conflict of interest.

Publisher's Note: All claims expressed in this article are solely those of the authors and do not necessarily represent those of their affiliated organizations, or those of the publisher, the editors and the reviewers. Any product that may be evaluated in this article, or claim that may be made by its manufacturer, is not guaranteed or endorsed by the publisher.

Copyright (C) 2022 Dai, Liu, Yuan, Li, Shi and Zhang. This is an open-access article distributed under the terms of the Creative Commons Attribution License (CC BY). The use, distribution or reproduction in other forums is permitted, provided the original author(s) and the copyright owner(s) are credited and that the original publication in this journal is cited, in accordance with accepted academic practice. No use, distribution or reproduction is permitted which does not comply with these terms. 